\title{
Short stature, platyspondyly, hip dysplasia, and retinal detachment: an atypical type II collagenopathy caused by a novel mutation in the $C$-propeptide region of COL2A1: a case report
}

\author{
Apiruk Sangsin 1,2,3,4, Chalurmpon Srichomthong ${ }^{1,2}$, Monnat Pongpanich ${ }^{5,6}$, Kanya Suphapeetiporn ${ }^{1,2,7^{*}}$ \\ and Vorasuk Shotelersuk ${ }^{1,2}$
}

\begin{abstract}
Background: Heterozygous mutations in COL2A1 create a spectrum of clinical entities called type II collagenopathies that range from in utero lethal to relatively mild conditions which become apparent only during adulthood. We aimed to characterize the clinical, radiological, and molecular features of a family with an atypical type II collagenopathy.

Case presentation: A family with three affected males in three generations was described. Prominent clinical findings included short stature with platyspondyly, flat midface and Pierre Robin sequence, severe dysplasia of the proximal femora, and severe retinopathy that could lead to blindness. By whole exome sequencing, a novel heterozygous deletion, c.4161_4165del, in COL2A1 was identified. The phenotype is atypical for those described for mutations in the C-propeptide region of COL2A1.

Conclusions: We have described an atypical type II collagenopathy caused by a novel out-of-frame deletion in the C-propeptide region of COL2A1. Of all the reported truncating mutations in the C-propeptide region that result in short-stature type II collagenopathies, this mutation is the farthest from the C-terminal of COL2A1.
\end{abstract}

Keywords: COL2A1, C-propeptide region, Exome sequencing, Type II collagenopathies

\section{Background}

Patients with COL2A1 mutations are collectively called type II collagenopathies. Missense mutations or in-frame derangement in the triple-helical region cause a phenotype on the spondyloepiphyseal dysplasia (SED) spectrum from lethal SED including achondrogenesis type II (ACG2; OMIM\# 200610) and hypochodrogenesis through spondyloepiphyseal dysplasia congenita (SEDC; OMIM\#183900), while mutations in the triple helical or N-propeptide regions cause Stickler syndrome type I (STL1; OMIM\# 108300) or Kniest dysplasia (OMIM\# 156550) [1]. Unlike

\footnotetext{
* Correspondence: kanya.Su@chula.ac.th

${ }^{1}$ Center of Excellence for Medical Genetics, Department of Pediatrics, Faculty

of Medicine, Chulalongkorn University, Bangkok 10330, Thailand

${ }^{2}$ Excellence Center for Medical Genetics, King Chulalongkorn Memorial

Hospital, the Thai Red Cross Society, 10330 Bangkok, Thailand

Full list of author information is available at the end of the article
}

mutations in the triple-helical or $\mathrm{N}$-propeptide regions, those in the $\mathrm{C}$-propeptide region generally produce atypical phenotypes such as platyspondylic lethal skeletal dysplasia, Torrance type (PLSDT\# OMIM 151210) [2], spondyloperipheral dysplasia (SPPD) (OMIM\# 271700) [3], vitreoretinopathy with phalangeal epiphyseal dysplasia (VPED) [4], avascular necrosis of the femoral head (ANFH; OMIM\# 608805) [5], or early-onset osteoarthritis (OA) [6]. Here, we describe a family with atypical features of type II collagenopathies caused by a novel mutation in COL2A1. As far as we know, this truncating mutation in the C-propeptide region is the farthest one from the 3' end of the gene that causes a disease with short stature, suggesting the existence of the mutant protein. 


\section{Case presentation \\ Subjects}

We studied a Thai family with skeletal dysplasia who attended the Genetics Clinic at the King Chulalongkorn Memorial Hospital, Bangkok, Thailand. The medical data, pedigree, physical examinations, and laboratory results were recorded. The written informed consent and parental consent (for the proband) was obtained after explanation of the possible consequences of this study.

\section{Genomic DNA preparation and whole-exome sequencing}

To perform genetic analysis, genomic DNA was isolated from peripheral blood leukocytes using a Puregene Blood kit (Qiagen, Hilden, Germany). The genomic DNA was sent to Macrogen, Inc. (Seoul, South Korea) for wholeexome sequencing (WES). DNA was captured using a SureSelect Human All Exon version 4 kit (Agilent Technologies, Santa Clara, CA) and sequenced on a Hiseq2000 instrument. Base calling was performed and quality scores were analyzed using Real Time Analysis software version 1.7. Sequence reads were aligned against the University of California Santa Cruz human genome assembly hg19 using Burrows-Wheeler Alignment software (bio-bwa.sourceforge.net/). Single-nucleotide variants (SNVs) and insertions/deletions (Indels) were detected by SAMTOOLS (samtools.sourceforge.net/) and annotated against dbSNP \& the 1000 Genomes Project. After quality filtering, we looked for variants located in the coding regions of known skeletal dysplasia genes for all potential pathogenic SNVs and Indels. Variant calling exclusion criteria were (a) coverage $<10 \times$; (b) quality score $<20$; (c) minor allele frequency $\geq 1 \%$ in the 1000 Genomes Project; and (d) non-coding variants and synonymous exonic variants. The remaining variants were subsequently filtered out if they were present in our in-house database of 165 unrelated Thai exomes. The variants were confirmed by PCR and Sanger sequencing.

Existing SNVs or known pathogenic mutations were filtered out using the Human Gene Mutation Database (http://www.hgmd.cf.ac.uk/ac/index.php) and the Exome Aggregation Consortium database (exac.broadinstitute.org).

\section{Results}

A 20 month-old male (IV:3; Fig. 1) is the first child of a non-consanguineous couple. His mother had miscarriages in the first trimester of the two previous pregnancies. The causes of both miscarriages were unknown. He was born at term by normal delivery with a birth weight of 2,850 g $\left(10^{\text {th }}\right.$ centile $)$ and a length of $45 \mathrm{~cm}\left(<3^{\text {rd }}\right.$ centile, $\left.-4 \mathrm{SD}\right)$. Physical examination revealed short stature, flattened face, cleft palate, micrognathia, short neck, and umbilical hernia (Fig. 2a). A radiograph obtained at age 20 months showed oval-shaped vertebral bodies (Fig. 2b). Ossification of the femoral head was absent. Long bones showed short broad

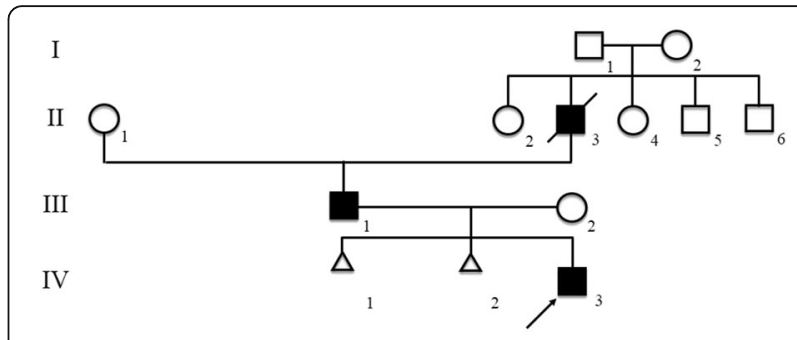

Fig. 1 Pedigree of the index family. The arrow indicates the proband

tubular shape with metaphyseal flaring (Fig. 2c). Other ossification centers appeared age-appropriate (Fig. 2c and d). Hands and feet radiographs were normal (Fig. 2e and f). His eyes examination and audiometry showed no abnormality. He was given a clinical diagnosis of SEDC.

The proband's father (III:1; Fig. 1) is a 26 year-old man. He had short trunk dwarfism with a height of $125 \mathrm{~cm}$ (-9 SD). He had a barrel-shaped chest, hyperlordosis of the lumbar spine, and flexion contracture of both hips (Fig. 3a). Hands and feet, including radiographs, were apparently normal (Fig. $3 \mathrm{~b}$ and c). A radiograph of the thoracolumbar spine showed flattened vertebral bodies with kyphotic deformity (Fig. 3d). Severe dysplasia of the bilateral proximal femoral epiphyses and hip dislocation were observed (Fig. 3e). Generalized osteopenia was noted. He was blind in the left eye since he was 8 years old, while his right eye had severe myopia and retinal detachment. He also had umbilical hernia when he was young but it spontaneously resolved.

The proband's grandfather (II:3; Fig. 1) had short stature $(-8 \mathrm{SD})$. He also had a barrel-shaped chest, hyperlordosis of the lumbar spine and flexion contracture of both hips. A radiograph of the thoracolumbar spine and pelvis showed flattened vertebral bodies, severe dysplasia of the bilateral proximal femoral epiphysis and hip dislocation (Fig. 3f). His hands and feet were normal. His eyes were reported normal but had never been formally evaluated. He died at the age of 54 because of septicemia after a nephrostomy as treatment for obstructive uropathy from ureteric stones. A blood sample was not available for mutation analysis.

Whole-exome sequencing (WES) of the proband revealed a five-nucleotide out-of-frame deletion (NM_001844.4: c.4161_4165del:p.Gln1387Hisfs"30) in COL2A1. PCR and Sanger sequencing using leukocyte-derived DNA from the proband (IV:3) and his father (III:1) confirmed that both were heterozygous for the mutation (Fig. 4a). This mutation has not been reported previously in the Human Genome Mutation Database or the Exome Aggregation Consortium database. In addition, it was not present in our in-house exome database of 165 Thai individuals. 

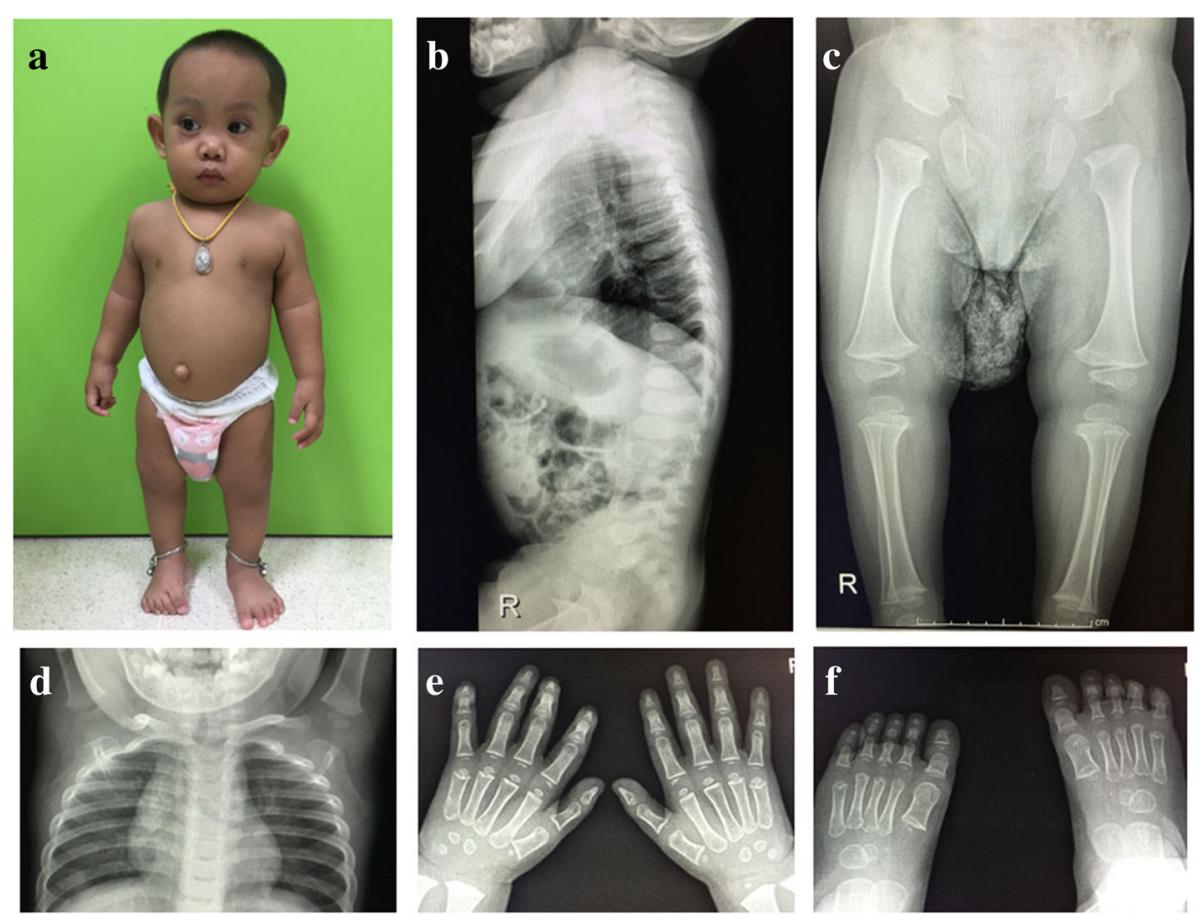

Fig. 2 a Photograph of the proband (IV: 3) showing flattened facial profile, micrognathia, short neck, and short trunk. b Whole spine lateral radiograph showing oval-shaped vertebral bodies. c Pelvis, femur, and tibial AP radiograph showing retarded ossification of the femoral heads while other ossification centers are age-appropriate. $\mathbf{d}$ Chest radiograph showing age-appropriate ossification centers at humeral heads and greater tuberosities of humeri. $\mathbf{e}, \mathbf{f}$ Hand and foot AP radiographs are normal
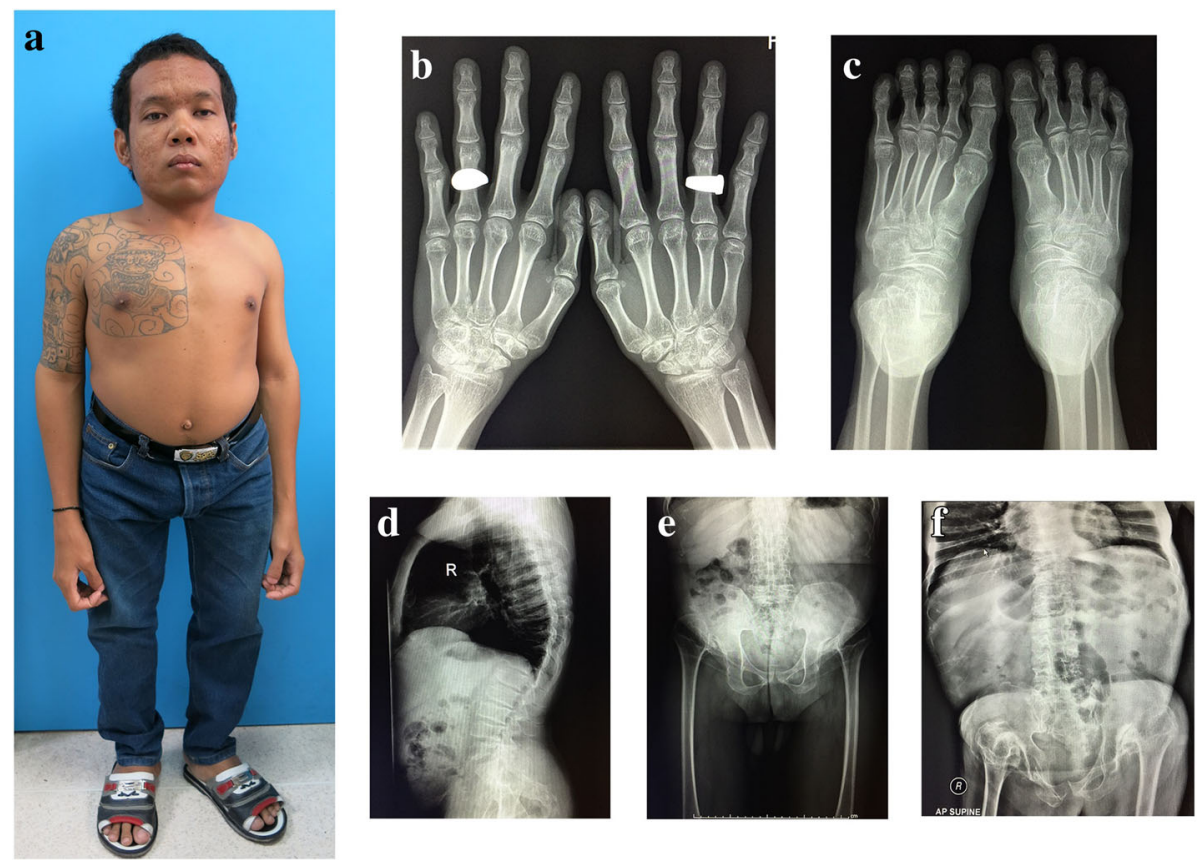

Fig. 3 a Photograph of proband's father (III: 1) showing disproportionate short stature and barrel-shaped chest. b, c Hand and feet radiographs are normal without brachydactyly. $\mathbf{d}$ Lateral radiograph of the thoracolumbar spine shows platyspondyly with kyphotic deformity. e Pelvis AP radiograph showing severe hip dysplasia and dislocation. $\mathbf{f}$ Thoracolumbar spine and pelvis AP radiographs of the proband's grandfather also show platyspondyly and severe hip dysplasia and dislocation 


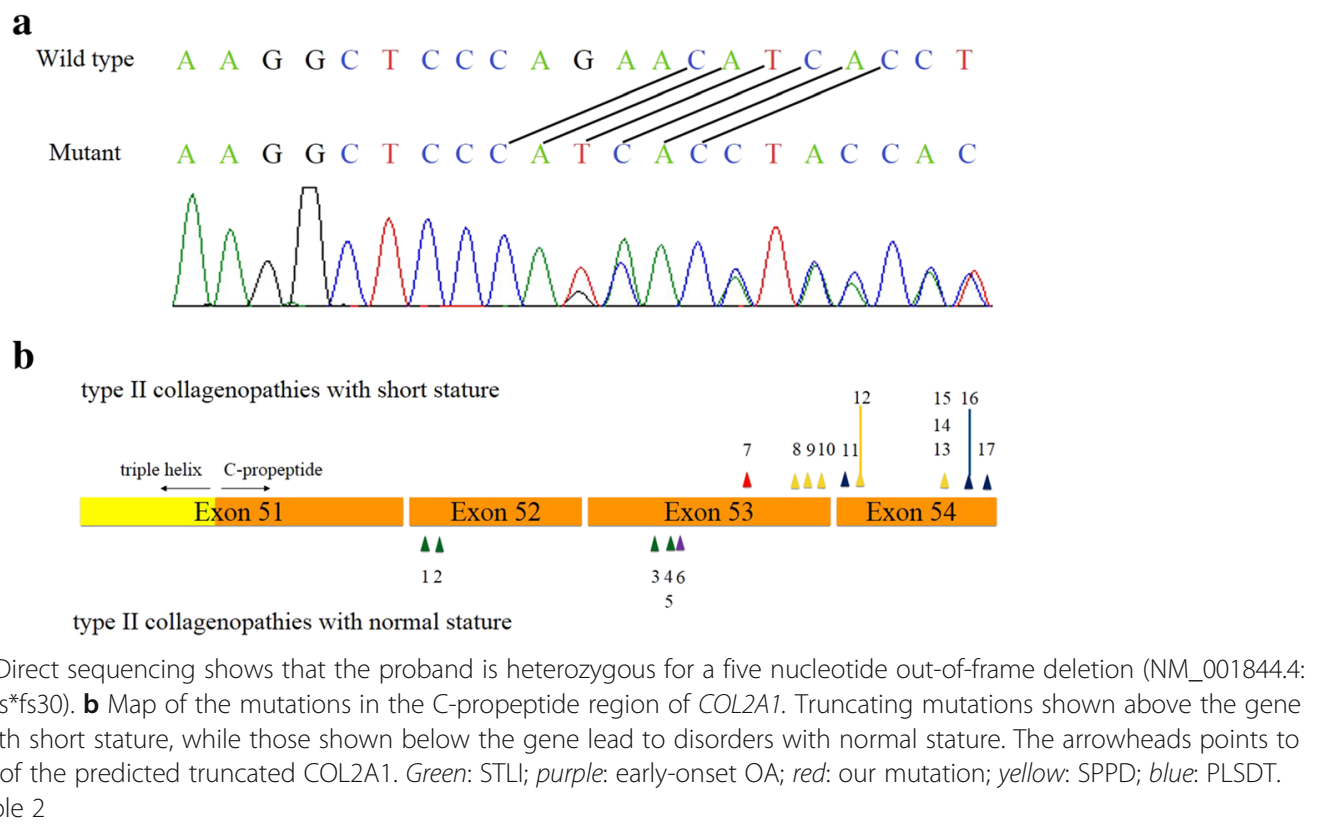

\section{Conclusions}

Major clinical and radiographic features in our patients include short stature with platyspondyly, flat midface, Pierre Robin sequence, hip dysplasia, and retinal detachment. The major extracellular structural protein of these affected organs is collagen type II. This led us to hypothesize that the etiologic mutation was in COL2A1. WES identified a five-base pair deletion in this gene. PCR and Sanger sequencing confirmed that the proband (IV:3) and his father (III:1) harbored a heterozygous mutation, c.4161_4165del, in COL2A1. This out-of-frame deletion is in the Cpropeptide region and is expected to lead to a protein truncation, p.Gln1387Hisfs*30.

Although our patients have a mutation in Cpropeptide region of COL2A1, the phenotype is atypical for those described for the mutations in C-propeptide region. Previous reported mutations in the C-propeptide region of collagen type II lead to one of the six entities: PLSDT, SPPD, VPED, ANFH, STL1, or early-onset OA. However, the clinical features of this family are different from all these six diseases (Table 1).

PLSDT was excluded by absence of wafer-thin platyspondyly, small round scapulae, and brachydactyly in our patients. Moreover, PLSDT patients generally die at birth [7] but our patients live into adulthood. An important diagnostic feature of SPPD, brachydactyly [3], excluded the diagnosis of SPPD. Short stature with platyspondyly without phalangeal epiphyseal dysplasia excluded VPED from the diagnosis [4]. ANFH patients have flattened femoral heads with signs of premature osteoarthritis. However, the femoral heads of proband's father (III:1) and grandfather (II: 3) were totally absent causing bilateral hip dysplasia and dislocation. Another important difference is the fact that ANFH patients do not have short stature, platyspondyly or retinal abnormalities [5], while our patients do. Patients with STLI usually have normal stature and premature OA of many joints [8,9], while all our patients (III:1 and II:3) had short stature ( -8 to -9 SD at adulthood). Early-onset OA patients should present with premature OA of joints without other skeletal abnormalities, while our patients had short stature and severe dysplasia of the hips.

STL1 is caused by haploinsufficiency of COL2A1. All point mutations in COL2A1 leading to STL1 are therefore expected to be subjected to nonsense-mediated mRNA decay (NMD). Heterozygous mutations leading to type II collagenopathies besides STL1 are predicted to have a dominant negative effect. Previous reports showed that truncating mutations in the $\mathrm{C}$-propeptide region led either to STL1 or SPPD, when the mutant RNA did, or did not, undergo NMD, respectively (Table 2).

Interestingly, our frameshift mutation, expected to create a stop codon 69 nucleotides upstream from the exon 53-54 junction, does not lead to STL1. We therefore hypothesize that the mutant RNA transcribed from the mutant COL2A1 allele found in our patients may not undergo NMD; instead that it may produce a mutant COL2A1 protein that has a dominant negative effect, interfering with the wild-type COL2A1 and leading to the unique phenotype. In general, mRNAs harboring a premature terminal codon (PCT) 50-55 nucleotides upstream of the last exon-exon junction are efficiency degraded [10]. The NMD is signaled by the presence of the exon junction complex (EJC). However, EJCs are not 
Table 1 Clinical features of type II collagenopathies resulting from mutations in the C-propeptide region of COL2A1, COmpared with this family

\begin{tabular}{|c|c|c|c|c|c|c|c|c|c|}
\hline & PLSDT & SPPD & VPED & ANFH & STLI & Early-onset OA & Proband (IV:3) & Father (III:1) & Grandfather (II:3) \\
\hline Lethal & + & - & - & - & - & - & - & - & - \\
\hline Short stature & + & + & - & - & - & N/A & + & + & + \\
\hline Delayed ossification & + & + & - & N/A & - & N/A & $\begin{array}{l}+ \\
\text { (hip only) }\end{array}$ & N/A & N/A \\
\hline Platyspondyly & + & + & - & - & + & N/A & + & + & + \\
\hline Metaphyseal involvement & + & + & - & - & - & N/A & + & + & N/A \\
\hline Epiphyseal involvement & + & + & + & $\begin{array}{l}+ \\
\text { (hip only) }\end{array}$ & + & N/A & + & + & + \\
\hline Early onset arthritis & N/A & + & + & $\begin{array}{l}+ \\
\text { (hip only) }\end{array}$ & + & + & N/A & - & N/A \\
\hline Brachydactyly & + & + & + & - & - & N/A & - & - & - \\
\hline Osteopenia/osteoporosis & N/A & N/A & N/A & + & + & N/A & N/A & + & + \\
\hline Early onset myopia & - & + & - & - & + & N/A & - & + & N/A \\
\hline Retinal detachment/tear & - & + & + & - & + & N/A & - & + & N/A \\
\hline Glaucoma & - & - & - & - & + & N/A & - & + & N/A \\
\hline Vitreal abnormalities & + & + & + & - & + & N/A & - & - & N/A \\
\hline Hearing loss & - & + & - & - & + & N/A & - & - & - \\
\hline Flattened facial profile & + & + & - & - & + & N/A & + & - & - \\
\hline Pierre-Robin sequence & + & N/A & - & - & + & N/A & + & - & - \\
\hline Cleft lip/cleft palate/bifid uvula & - & + & - & - & + & N/A & + & - & - \\
\hline
\end{tabular}

N/A not available, PLSDT platyspondylic lethal skeletal dysplasia, Torrance type, SPPD spondyloperipheral dysplasia, VPED vitreoretinopathy with phalangeal epiphyseal dysplasia, ANFH avascular necrosis of the femoral head, STL1 Stickler syndrome type I, OA osteoarthritis

Table 2 Truncating mutations in the C-propeptide region of COL2A1

\begin{tabular}{|c|c|c|c|c|c|}
\hline No. & Variant location & Nucleotide change & Predicted protein change & Clinical diagnosis & Reference \\
\hline 1 & Exon 52 & c.3906del & p.Asn1303Thrfs*9 & STL1 & Hoornaert et al. [12] \\
\hline 2 & Exon 52 & c.3891_3898dup & p.lle1300Thrsfs*15 & STL1 & Hoornaert et al. [12] \\
\hline 3 & Intron 52 & $c .4074+1 G>T$ & p.Trp1348Cysfs*17 & STLI & Hoornaert et al. [12] \\
\hline 4 & Exon 52 & c.3978delC & p.Asn1327llefs*49 & STLI & Ahmad et al. [13] \\
\hline 5 & Exon 52 & c.3957del & p.Gly1320Alafs*56 & STL1 & Annunen et al. [14] \\
\hline 6 & Exon 53 & c.4088del & p.Asp1363Valfs*13 & Early-onset $\mathrm{OA}$ & Barat-Houari et al. [15] \\
\hline 7 & Exon 53 & c.4161_4165del & p.Gln1387Hisfs*30 & This report & \\
\hline 8 & Exon 53 & c.4300del & p.Leu1434* & SPPD & Barat-Houari et al. [15] \\
\hline 9 & Exon 53 & c.4287_4291dup & p.Tyr1431Serfs*6 & SPPD & Zabel et al. [16] \\
\hline 10 & Exon 53 & c. $4314 C>A$ & p.Cys $1438^{*}$ & SPPD & Zankl et al. [3] \\
\hline 11 & Exon 54 & c. $4335 \mathrm{G}>\mathrm{A}$ & p.Trp $1445^{*}$ & PLSDT & Zankl et al. [2] \\
\hline 12 & Exon 54 & c. $4339 \mathrm{~A}>\mathrm{T}$ & p.Lys $1447^{*}$ & SPPD & Bedeschi et al. [17] \\
\hline 13 & Exon 54 & c.4332del & p.Lys 1444 Asnfs*27 & SPPD & Zhang et al. [18] \\
\hline 14 & Exon 54 & c.4337del & p.Gly1446Alafs*25 & SPPD & Zankl et al. [3] \\
\hline 15 & Exon 54 & c.4357del & p.Arg1453Glyfs*18 & SPPD & Meredith et al. [19] \\
\hline 16 & Exon 54 & c. $.4423 C>T$ & p.Gln1475* & PLSDT & Zankl et al. [2] \\
\hline 17 & Exon 54 & c.4413_4416del & p.Gly 1472 Profs $^{* 9}$ & PLSDT & Nishimura et al. [20] \\
\hline
\end{tabular}


equally assembled at every exon junction. Therefore, it is possible that the variation of the EJC may affect NMD efficiency and lead to efficient degradation of COL2A1 mRNAs harboring a PTC at least 70 nucleotides upstream of the final exon-exon junction. On the contrary, mutations causing premature stop codons from 69 nucleotides upstream of the exon 53-54 junction down to the 3' end COL2A1 would probably not undergo NMD, have a dominant negative effect, and therefore cause more severe phenotypes of the spectrum such as SPPD and PLSDT $[2,3]$. Unfortunately, tissues from our patients that would express COL2A1 are not available to test this hypothesis. Of all the reported mutations in the C-propeptide region that result in short-stature type II collagenopathies, our mutation is the farthest truncating mutation from the C-terminal of COL2A1 (Fig. 4b, Table 2).

Our probands and his father had some different phenotypes such as facial profile, eye involvement, and cleft palate. Intra-and extrafamilial variability of type II collagenopathies has previously been observed in patients with the same mutation. For instance, in a family with VPED, a 22-year-old patient had severe premature osteoarthritis requiring hip replacement while two older siblings had only mild osteoarthritis [4]. In another family, while the mosaic mother had SPPD, her fetus suffered from the lethal PLSDT [11]. These data suggest that genetic, epigenetic, and environmental modifiers affect the clinical presentation in type II collagenopathies. Moreover, these modifiers may be other possible explanations for the atypical phenotype of the patients.

In conclusion, we report an atypical phenotype resulting from a novel truncating mutation in the $\mathrm{C}$ propeptide region of $C O L 2 A 1$ with prominent features including short stature, platyspondyly, hip dysplasia, and retinal detachment.

\section{Abbreviations \\ ACG2: Achondrogenesis type II; ANFH: Avascular necrosis of the femoral head; NMD: Nonsense-mediated mRNA decay.; OA: Osteoarthritis; PLSDT: Platyspondylic lethal skeletal dysplasia; SED: Spondyloepiphyseal dysplasia; SEDC: Spondyloepiphyseal epiphyseal dysplasia congenital; SPPD: Spondyloperipheral dysplasia; STL1: Stickler syndrome type I; VPED: Vitreoretinopathy with phalangeal epiphyseal dysplasia; WES: Whole-exome sequencing}

\section{Acknowledgements}

We thank the family members for participating in this study.

\section{Funding}

This study was supported by the Chulalongkorn Academic Advancement into Its 2nd Century Project and Thailand Research Fund.

\section{Availability of data and materials}

All data supporting our finding are included in the manuscript.

\section{Authors' contributions}

AS participated in the molecular genetic studies and wrote the manuscript, CS participated in molecular genetic studies, MP participated in molecular genetic studies, KS assured the general supervision of the research group, VS assured the general supervision of the research group and raised funding. All authors revised and approved the final version of the manuscript.

\section{Competing interest}

The authors declare that they have no competing interest.

\section{Consent for publication}

Consent for publication of the cases was obtained for each participant.

\section{Ethics approval and consent to participate}

Ethical approval was obtained from the institutional review board, Faculty of Medicine, Chulalongkorn University. After explanation of the possible consequences of this study, the written informed consent and parental consent (for the proband) was obtained.

\section{Author details}

${ }^{1}$ Center of Excellence for Medical Genetics, Department of Pediatrics, Faculty of Medicine, Chulalongkorn University, Bangkok 10330, Thailand. ${ }^{2}$ Excellence Center for Medical Genetics, King Chulalongkorn Memorial Hospital, the Thai Red Cross Society, 10330 Bangkok, Thailand. ${ }^{3}$ Department of Orthopedics, Faculty of Medicine, Chiang Mai University, 50200 Chiang Mai, Thailand. ${ }^{4}$ Interdepartment Program of Biomedical Sciences, Faculty of Graduate School, Chulalongkorn University, 10330 Bangkok, Thailand. ${ }^{5}$ Department of Mathematics and Computer Science, Faculty of Science, Chulalongkorn University, 10330 Bangkok, Thailand. ${ }^{6}$ Program in Bioinformatics and Computational Biology, Graduate School, Chulalongkorn University, 10330 Bangkok, Thailand. 'Division of Medical Genetics and Metabolism, Department of Pediatrics, Faculty of Medicine, Chulalongkorn University, Sor Kor Building 11th floor, 10330 Bangkok, Thailand.

Received: 31 May 2016 Accepted: 30 November 2016

Published online: 12 December 2016

\section{References}

1. Nishimura G, Haga N, Kitoh H, Tanaka Y, Sonoda T, Kitamura M, Shirahama S, Itoh T, Nakashima E, Ohashi H, et al. The phenotypic spectrum of COL2A1 mutations. Hum Mutat. 2005;26(1):36-43.

2. Zankl A, Neumann L, Ignatius J, Nikkels P, Schrander-Stumpel C, Mortier G, Omran $H$, Wright $M$, Hilbert $K$, Bonafe $L$, et al. Dominant negative mutations in the C-propeptide of COL2A1 cause platyspondylic lethal skeletal dysplasia, torrance type, and define a novel subfamily within the type 2 collagenopathies. Am J Med Genet A. 2005;133A(1):61-7.

3. Zankl A, Zabel B, Hilbert K, Wildhardt G, Cuenot S, Xavier B, Ha-Vinh R, Bonafe L, Spranger J, Superti-Furga A. Spondyloperipheral dysplasia is caused by truncating mutations in the C-propeptide of COL2A1. Am J Med Genet A. 2004;129A(2):144-8.

4. Richards AJ, Morgan J, Bearcroft PW, Pickering E, Owen MJ, Holmans P, Williams N, Tysoe C, Pope FM, Snead MP, et al. Vitreoretinopathy with phalangeal epiphyseal dysplasia, a type II collagenopathy resulting from a novel mutation in the C-propeptide region of the molecule. J Med Genet. 2002;39(9):661-5.

5. Kannu P, O'Rielly DD, Hyland JC, Kokko LA. Avascular necrosis of the femoral head due to a novel C propeptide mutation in COL2A1. Am J Med Genet A. 2011;155A(7):1759-62.

6. Barat-Houari M, Sarrabay G, Gatinois V, Fabre A, Dumont B, Genevieve D, Touitou I. Mutation update for COL2A1 gene variants associated with type II collagenopathies. Hum Mutat. 2016;37(1):7-15.

7. Horton WA, Rimoin DL, Hollister DW, Lachman RS. Further heterogeneity within lethal neonatal short-limbed dwarfism: the platyspondylic types. J Pediatr. 1979;94(5):736-42.

8. Letts M, Kabir A, Davidson D. The spinal manifestations of Stickler's syndrome. Spine. 1999;24(12):1260-4.

9. Rose PS, Levy HP, Liberfarb RM, Davis J, Szymko-Bennett Y, Rubin BI, Tsilou E, Griffith AJ, Francomano CA. Stickler syndrome: clinical characteristics and diagnostic criteria. Am J Med Genet A. 2005;138A(3):199-207.

10. Hug N, Longman D, Caceres JF. Mechanism and regulation of the nonsense-mediated decay pathway. Nucleic Acids Res. 2016;44(4):1483-95.

11. Desir J, Cassart M, Donner C, Coucke P, Abramowicz M, Mortier G. Spondyloperipheral dysplasia as the mosaic form of platyspondylic lethal skeletal dyplasia torrance type in mother and fetus with the same COL2A1 mutation. Am J Med Genet A. 2012;158A(8):1948-52. 
12. Hoornaert KP, Marik I, Kozlowski K, Cole T, Le Merrer M, Leroy JG, Coucke PJ, Sillence D, Mortier GR. Czech dysplasia metatarsal type: another type II collagen disorder. Eur J Hum Genet 2007, 15(12):1269-1275.

13. Ahmad NN, Dimascio J, Knowlton RG, Tasman WS. Stickler syndrome. A mutation in the nonhelical $3^{\prime}$ end of type II procollagen gene. Arch Ophthalmol 1995, 113(11):1454-1457.

14. Annunen S, Korkko J, Czarny M, Warman ML, Brunner HG, Kaariainen H, Mulliken JB, Tranebjaerg L, Brooks DG, Cox GF et al. Splicing mutations of 54-bp exons in the COL11A1 gene cause Marshall syndrome, but other mutations cause overlapping Marshall/Stickler phenotypes. Am J Hum Genet 1999, 65(4):974-983.

15. Barat-Houari M, Dumont B, Fabre A, Them FT, Alembik Y, Alessandri JL, Amiel J, Audebert S, Baumann-Morel C, Blanchet P et al. The expanding spectrum of COL2A1 gene variants IN 136 patients with a skeletal dysplasia phenotype. Eur J Hum Genet 2016, 24(7):992-1000

16. Zabel B, Hilbert K, Stoss H, Superti-Furga A, Spranger J, Winterpacht A. A specific collagen type II gene (COL2A1) mutation presenting as spondyloperipheral dysplasia. Am J Med Genet 1996, 63(1):123-128.

17. Bedeschi MF, Bianchi V, Gentilin B, Colombo L, Natacci F, Giglio S, Andreucci E, Trespidi L, Acaia B, Furga AS et al. Prenatal manifestation and management of a mother and child affected by spondyloperipheral dysplasia with a C-propeptide mutation in COL2A1: case report. Orphanet J Rare Dis 2011, 6:7.

18. Zhang Z, Zhao SC, He JW, Fu WZ, Zhang CQ, Zhang ZL. Identification of one novel mutation in the C-propeptide of COL2A1 in a Chinese family with spondyloperipheral dysplasia. Gene 2013, 522(1):107-110.

19. Meredith SP, Richards AJ, Bearcroft P, Pouson AV, Snead MP. Significant ocular findings are a feature of heritable bone dysplasias resulting from defects in type II collagen. Brit J Ophth 2007, 91(9):1148-1151.

20. Nishimura G, Nakashima E, Mabuchi A, Shimamoto K, Shimamoto T, Shimao Y, Nagai T, Yamaguchi T, Kosaki R, Ohashi H et al. Identification of COL2A1 mutations in platyspondylic skeletal dysplasia, Torrance type. J Med Genet 2004, 41(1):75-79.

\section{Submit your next manuscript to BioMed Central and we will help you at every step:}

- We accept pre-submission inquiries

- Our selector tool helps you to find the most relevant journal

- We provide round the clock customer support

- Convenient online submission

- Thorough peer review

- Inclusion in PubMed and all major indexing services

- Maximum visibility for your research

Submit your manuscript at www.biomedcentral.com/submit

) Biomed Central 